\title{
Mathematical Analysis of an Epidemic-Species Hybrid Dynamical System
}

\author{
Jing Hui, Jian-Hua Pang, and Dong-Rong Lin \\ Department of Science and Technology, Guangxi University of Science and Technology, Liuzhou 545006, China \\ Correspondence should be addressed to Jing Hui; jw@gxut.edu.cn
}

Received 9 March 2014; Revised 30 June 2014; Accepted 20 July 2014; Published 28 August 2014

Academic Editor: Hai-Feng Huo

Copyright (C) 2014 Jing Hui et al. This is an open access article distributed under the Creative Commons Attribution License, which permits unrestricted use, distribution, and reproduction in any medium, provided the original work is properly cited.

\begin{abstract}
We consider an epidemic-species hybrid dynamical system. The disease is spread among the prey only and the infected prey can reproduce virus. The predator only eats the infected prey. Mathematical analyses are given for the system with regard to the existence of equilibria, local stability, Hopf bifurcation, and the orbital stability of the Hopf bifurcating limit cycle. We further analyse the system under impulsive releasing of virus and predator.
\end{abstract}

\section{Introduction}

Epidemic models and species models have received much attention from scientists, respectively. There are many literatures about them. Now we consider an epidemic-species hybrid dynamical system which is motivated by the integrated pest management (IPM). As we know, IPM is becoming more and more popular among farmers, researchers, and policy makers. IPM seeks to minimize reliance on pesticides by emphasizing the contribution of other control methods, including biological control, host-plant resistance breeding, and cultural techniques. Potentially, the use of viruses, fungi, and bacteria is one of the most effective biological methods for controlling pests. One reason for this optimism is that the generation time lapse of microbes is much shorter than that of an insect pest [1]. The role of microbial pesticides in the integrated management of insect pests has been recently reviewed for agriculture [2-5], forestry [6,7], and public health [8]. In most cases no single microbial control agent will provide sustainable control of an insect pest or complex of pests. As components of an integrated approach, entomopathogens can provide significant and selective insect control. In the not too distant future we can envision a broader appreciation for the attributes of entomopathogens and expect to see synergistic combinations of microbial control agents with other technologies (in combination with semichemical technology, soft chemical pesticides, other natural enemies, resistant plants, chemigation, remote sensing, etc.) that will enhance the effectiveness and sustainability of integrated control strategies.

There is a vast amount of literature on the applications of microbial disease to suppress pests $[9,10]$. But there are only a few papers on mathematical models of the dynamics of microbial disease in pest control [11, 12]. On the other hand, there are many mathematical models of infectious diseases of human beings [13-16]. All the earlier pest control models are normally two-dimensional consisting of susceptible and infected pests, and infectious disease models are at most three-dimensional with a susceptible, infected, and removable class; the dynamics of the diseasescausing organism is not considered in the model. However, presently the insect pathogens are used in two ways [17]. In the first method, a small amount of pathogens is introduced in the pest population with the expectation that it will generate an epidemic and will be subsequently endemic. The success of the approach depends on the survival of the microbes which in turn depends on environmental factors, such as temperature, humidity, and crop conditions. In the second method, an insect pathogen is used like a nonresidual chemical insecticide. In this case, it is applied whenever a pest population is at an economically extreme level for pest damage and there is no expectation that the pathogen will survive for an appreciable period. In this paper, we examine the use of pathogens in a four-dimensional prey-predator model for the agricultural or forestry ecosystem with the 
assumption that viral disease spreads only among the insect pests. In nature, the infection by baculovirus begins when an insect eats virus particles on a plant, perhaps from a sprayed treatment. Virus infection causes the cell lysis in the host body and produces more virus particles (virus replication), until the cell and ultimately the insect die. Most baculoviruses cause the host insect to die in a way that maximizes the chance that other insects come in contact with the virus and become infected by eating the foliage that has been contaminated by virus-killed larvae. We further assume that the natural enemy (predator) in the system survives on the infected prey. This is due to the fact that the viral infection makes some behavioral changes and sublethal effects on host, which make them more vulnerable to predation by natural enemies than healthy hosts before death [18].

The dynamical behavior of the considered system is investigated from the point of view of stability and persistence. The model shows that infection can be sustained only above a threshold force of infection resulting from virus replication parameter $k$. Still, on increasing the value of $k$, the endemic equilibrium bifurcates towards a periodic solution. The stability of the limit cycles arising from Hopf bifurcation is analysed using Poore's condition [19]. Whereas, in consideration of the practice in pest control, the natural enemy and the pathogens are introduced discontinuously at some fixed moment, the impulsive releasing of the virus and natural enemy is added in the system. We prove that the pest extinction periodic solution exists and is globally asymptotically stable when the impulsive period $\tau$ is less than the critical value; otherwise, the system can be permanent. The arrangement of the paper is as follows. In Section 2 we describe the model formulation. Section 3 shows its equilibria. Section 4 discusses the local stability and Hopf bifurcation. In Section 5 we give the details about the orbital stability of the periodic orbit arising from Hopf bifurcation by Poore's condition. In Section 6 we consider the corresponding impulsive system by releasing virus and natural enemy at some fixed moment. Lastly in Section 7 we end the paper with a concluding discussion.

\section{The Model}

The model contains three species, namely, pest, predator, and virus. The total pest population $N$ is divided into two subpopulation classes: the susceptible pest, denoted by $S$, and the virus-infected pest, denoted by $I$. Therefore, at any time $t, N(t)=S(t)+I(t)$. We assume that only susceptible pest $S$ is capable of reproducing with logistic law; that is, the infected class of pest is removed by lysis before having the possibility of reproducing. However, they still contribute with $S$ to population growth towards the carrying capacity $K . r$ is the intrinsic birth rate. Then the growth equation of susceptible pest $S$ is given by

$$
\frac{d S}{d t}=r S\left(1-\frac{S+I}{K}\right)
$$

A susceptible pest becomes infected as the two mentioned methods ahead. The incidence is assumed to be the simple mass action incidence $\lambda S V ; \lambda$ represents the effective per pest contact rate with viruses. Hence, the evolution equation of the susceptible pest is

$$
\frac{d S}{d t}=r S\left(1-\frac{S+I}{K}\right)-\lambda S V .
$$

The infected pest $I$ has a latent period, the period between the instant of infection and that of lysis, during which the virus is reproduced inside the larval tissue. The lysis death rate $\xi$ gives a measure of such latency period $T$, given by $T=1 / \xi$. The equation of infected class $I$ takes the form

$$
\frac{d I}{d t}=\lambda S V-\xi I .
$$

The lysis largely produces virus polyhedra or polyhedral inclusion bodies (PIB) on average $k$ PIB per insect; $k(k>1)$ is called the virus replication parameter. The virus particles $V$ have natural mortality denoted by $\mu_{v}$ due to temperature changes, enzymatic attack, $\mathrm{pH}$ dependence, and so forth. The equation of the virus is given by

$$
\frac{d V}{d t}=-\mu_{v} V+k \xi I
$$

We assume the natural enemy or predator $P$ consumes only the infected prey $I$, the consuming means is the standard bilinear expression $l I P$, and $l$ is the predation coefficient. We assume the natural enemies in the system remain unharmed from the virus. Then the equations of the infected pest and the natural enemy are

$$
\begin{gathered}
\frac{d I}{d t}=\lambda S V-l I P-\xi I, \\
\frac{d P}{d t}=P\left(-d-\epsilon_{P} P+C l I\right),
\end{gathered}
$$

where $d$ is the death rate of the predator, $\epsilon_{P}$ is the density restriction coefficient of predator, and $C$ is the conversion factor for the predator. Keeping these in view, the dynamics of the epidemic-species may be governed by the following autonomous system of differential equations:

$$
\begin{gathered}
\frac{d S}{d t}=r S\left(1-\frac{S+I}{K}\right)-\lambda S V, \\
\frac{d I}{d t}=\lambda S V-l I P-\xi I, \\
\frac{d P}{d t}=P\left(-d-\epsilon_{P} P+C l I\right), \\
\frac{d V}{d t}=-\mu_{v} V+k \xi I .
\end{gathered}
$$

In the above model, all of the coefficients, $r, K, \lambda, l, \xi, d, \epsilon_{P}$, $C, \mu_{v}$, and $k$, are positive real numbers.

If using the dimensionless time $\tau=\lambda K t$ and the transformations $s=S / K, i=I / K, p=l P / K$, and $v=$ $V / K$, we have the dimensionless form of model (6). For 
convenience, we still write $t$ in place of $\tau$; the corresponding dimensionless form of model (6) is given as follows:

$$
\begin{gathered}
\frac{d s}{d t}=a s(1-s-i)-s v, \\
\frac{d i}{d t}=s v-\frac{1}{\lambda} i p-\eta i, \\
\frac{d p}{d t}=p(-d-\epsilon p+c i), \\
\frac{d v}{d t}=-\mu v+k \eta i,
\end{gathered}
$$

where we denote $a=r /(\lambda K), \eta=\xi /(\lambda K), \epsilon=\epsilon_{P} /(\lambda l), c=$ $(C l) / \lambda$, and $\mu=\mu_{v} /(\lambda K)$.

\section{Equilibria of System (7)}

Equilibria of model (7) are obtained by solving $d s / d t=$ $d i / d t=d p / d t=d v / d t=0$. It can be checked that model (7) has the following three boundary equilibria: the vanishing equilibrium $E_{0}(0,0,0,0)$; the disease-free equilibrium $E_{1}(1,0,0,0)$; and the predator-free equilibrium $E_{2}(\bar{s}, \bar{i}, 0, \bar{v})$, where $\bar{s}=\mu / k, \bar{i}=a \mu(k-\mu) / k(k \eta+a \mu)$, and $\bar{v}=$ $a \eta(k-\mu) /(k \eta+a \mu)$; when $k>\mu$, the equilibrium $E_{2}$ is nonnegative. The positive equilibrium $E^{*}\left(s^{*}, i^{*}, p^{*}, v^{*}\right)$ is obtained by solving the following equations:

$$
\begin{gathered}
a\left(1-s^{*}-i^{*}\right)-v^{*}=0, \\
s^{*} v^{*}-\frac{1}{\lambda} i^{*} p^{*}-\eta i^{*}=0, \\
-d-\epsilon p^{*}+c i^{*}=0, \\
-\mu v^{*}+k \eta i^{*}=0 .
\end{gathered}
$$

From the fourth equation of the above equations, we have $v^{*}=(k \eta / \mu) i^{*}$; substituting it into the first equation, then $s^{*}=1-(1+k \eta / a \mu) i^{*}$; from the third equation of $(8)$, $p^{*}=\left(-d+c i^{*}\right) / \epsilon$; substituting $s^{*}, v^{*}$, and $p^{*}$ into the second equation, we know the infected pest satisfies the following equation: $-\left(k \eta / \mu+k^{2} \eta^{2} /\left(a \mu^{2}\right)+c /(\lambda \epsilon)\right)\left(i^{*}\right)^{2}+(k \eta / \mu+d /(\lambda \epsilon)-$ $\eta) i^{*}=0$, so $i^{*}=\left(\eta a \mu \lambda \epsilon(k-\mu)+a d \mu^{2}\right) /\left(k \eta a \mu \lambda \epsilon+k^{2} \eta^{2} \lambda \epsilon+\right.$ $\left.a c \mu^{2}\right)$. For the positivity of $E^{*}$, we assume $k>\mu$ and $d / c<$ $\left(\eta a \mu \lambda \epsilon(k-\mu)+a d \mu^{2}\right) /\left(k \eta a \mu \lambda \epsilon+k^{2} \eta^{2} \lambda \epsilon+a c \mu^{2}\right)<a \mu /(a \mu+$ $k \eta)$. If $k<\mu$, then the predator-free equilibrium $E_{2}$ and the positive equilibrium $E^{*}$ do not exist, which implies the virus replication parameter $k$ is too small to support the virus invasion in insect pest. When the virus replication parameter $k$ is larger than $\mu$ and very close to $\mu$, then the predator-free equilibrium $E_{2}$ will collapse to the disease-free equilibrium $E_{1}$. Thus it can be seen that the virus replication parameter $k$ plays an important role for the dynamics of system (7). In summary, we have the following theorem about the equilibria of system (7).

Theorem 1. System (7) always has two equilibria, namely, the vanishing equilibrium $E_{0}(0,0,0,0)$ and the disease-free equilibrium $E_{1}(1,0,0,0)$; if $k>\mu$, there is one more predatorfree equilibrium $E_{2}(\bar{s}, \bar{i}, 0, \bar{v})$; further, if the virus replication parameter satisfies $k>\mu$ and $d / c<(\eta a \mu \lambda \epsilon(k-\mu)+$ $\left.a d \mu^{2}\right) /\left(k \eta a \mu \lambda \epsilon+k^{2} \eta^{2} \lambda \epsilon+a c \mu^{2}\right)<a \mu /(a \mu+k \eta)$, then the positive equilibrium $E^{*}$ exists.

\section{Local Stability and Hopf Bifurcation}

The dynamical behaviour of equilibria can be studied by computing variational matrices corresponding to each equilibrium. The variational matrix of (7) is

$$
J=\left[\begin{array}{cccc}
a-2 a s-a i-v & -a s & 0 & -s \\
v & -\frac{1}{\lambda} p-\eta & -\frac{1}{\lambda} i & s \\
0 & c p & -d-2 \epsilon p+c i & 0 \\
0 & k \eta & 0 & -\mu
\end{array}\right] .
$$

For the vanishing equilibrium $E_{0}$, correspondingly, variational matrix is

$$
J_{E_{0}}=\left[\begin{array}{cccc}
a & 0 & 0 & 0 \\
0 & -\eta & 0 & 0 \\
0 & 0 & -d & 0 \\
0 & k \eta & 0 & -\mu
\end{array}\right]
$$

Obviously, $E_{0}$ is unstable saddle point with threedimensional stable manifold and one-dimensional unstable manifold.

For the disease-free equilibrium $E_{1}$, the corresponding variational matrix is

$$
J_{E_{1}}=\left[\begin{array}{cccc}
-a & -a & 0 & -1 \\
0 & -\eta & 0 & 1 \\
0 & 0 & -d & 0 \\
0 & k \eta & 0 & -\mu
\end{array}\right]
$$

The characteristic equation is $(\Lambda+a)(\Lambda+d)[(\Lambda+\eta)(\Lambda+\mu)-$ $k \eta]=0$; that is, $(\Lambda+a)(\Lambda+d)\left[\Lambda^{2}+(\mu+\eta) \Lambda-\eta(k-\mu)\right]=0$. When $1<k<\mu$, there are four real negative eigenvalues and consequently the disease-free equilibrium $E_{1}$ is locally asymptotically stable; when $k=\mu$, corresponding to the disease-free equilibrium $E_{1}$, there are a simple eigenvalue 0 and another three negative eigenvalues. The system (7) enters into a saddle-node bifurcation at $E_{1}$; when $k>\mu$, there are three real negative eigenvalues and one positive eigenvalue so the disease-free equilibrium $E_{1}$ is unstable.

For the predator-free equilibrium $E_{2}$, the corresponding variational matrix is

$$
J_{E_{2}}=\left[\begin{array}{cccc}
-a \bar{s} & -a \bar{s} & 0 & -\bar{s} \\
\bar{v} & -\eta & -\frac{1}{\lambda} \bar{i} & \bar{s} \\
0 & 0 & -d+c \bar{i} & 0 \\
0 & k \eta & 0 & -\mu
\end{array}\right] .
$$

The characteristic equation is $(\Lambda+d-c \bar{i})\left(\Lambda^{3}+d_{1} \Lambda^{2}+d_{2} \Lambda+\right.$ $\left.d_{3}\right)=0$, where $d_{1}=\mu+\eta+a \bar{s}, d_{2}=a \bar{s}(\mu+\eta+\bar{v})$, and $d_{3}=a \eta \mu(1-\bar{s})$. The characteristic equation has clearly one negative real root, namely, $c \bar{i}-d$, and another three roots are given by the equation $\Lambda^{3}+d_{1} \Lambda^{2}+d_{2} \Lambda+d_{3}=0 . d_{1}, d_{2}$, and $d_{3}$ can be looked as the function of $\bar{s}$; since $\bar{s}=\mu / k$ and the predator-free equilibrium $E_{2}$ exists if $k>\mu, \bar{s} \in(0,1)$. 
Now we apply Routh-Hurwitz criteria and the definition of a simple criterion of Hopf bifurcation [20] to show the local stability of $E_{2}$ and the Hopf bifurcation at $E_{2}$. That is to say, for any $\bar{s} \in(0,1), E_{2}$ is locally asymptotically stable if and only if $d_{i}(\bar{s})>0, i=1,2,3$, and $H(\bar{s})=d_{1}(\bar{s}) d_{2}(\bar{s})-d_{3}(\bar{s})>0$. A Hopf bifurcation of the predator-free equilibrium $E_{2}$ occurs at $\bar{s}=\bar{s}_{0} \in(0,1)$ if and only if $H\left(\bar{s}_{0}\right)=0,\left.(d H / d \bar{s})\right|_{\bar{s}_{0}} \neq 0$.

In fact, $d_{i}(\bar{s})>0, i=1,2,3$, is obvious under the condition $k>\mu$. For the function $H(\bar{s}), \bar{s} \in(0,1)$, since $H(0)=-a \eta \mu<0$ and $H(1)=(\mu+\eta+a) a(\mu+\eta+\bar{v})>0$, based on the intermediate value theorem, there exists a $\bar{s}_{0} \in(0,1)$ so that $H\left(\bar{s}_{0}\right)=0$. In addition, $d H / d \bar{s}=a d_{2}(\bar{s})+d_{1}(\bar{s}) a(\mu+$ $\eta+\bar{v})+a \eta \mu>0$ and $d H / d \bar{s}>0$ at $\bar{s}=0$; at the same time $d^{2} H / d \bar{s}^{2}=2 a^{2}(\mu+\eta+\bar{v})>0$, so $\left.(d H / d \bar{s})\right|_{\bar{s}_{0}}>0$. Thus $H(\bar{s})>0$ at $\bar{s} \in\left(\bar{s}_{0}, 1\right)$ which implies that the predator-free equilibrium $E_{2}$ is locally asymptotically stable in $\left(\bar{s}_{0}, 1\right)$ and unstable in $\left(0, \bar{s}_{0}\right)$; further a Hopf bifurcation of the predatorfree equilibrium $E_{2}$ occurs at $\bar{s}=\bar{s}_{0} \in(0,1)$; for decreasing $\bar{s} \in\left(0, \bar{s}_{0}\right)$, it approaches a periodic solution, whose period is $2 \pi / \omega_{0}$, where $\omega_{0}=\sqrt{d_{2}\left(\bar{s}_{0}\right)}=\sqrt{d_{3}\left(\bar{s}_{0}\right) / d_{1}\left(\bar{s}_{0}\right)}$.

For the positive equilibrium $E^{*}$, the corresponding variational matrix is

$$
J_{E^{*}}=\left[\begin{array}{cccc}
-a s^{*} & -a s^{*} & 0 & -s^{*} \\
v^{*} & -\frac{1}{\lambda} p^{*}-\eta & -\frac{1}{\lambda} i^{*} & s^{*} \\
0 & c p^{*} & -\epsilon p^{*} & 0 \\
0 & k \eta & 0 & -\mu
\end{array}\right]
$$

For the local stability of positive equilibrium $E^{*}$, we have the following theorem.

Theorem 2. The positive equilibrium $E^{*}$ is locally asymptotically stable if $k<\left(\mu / \eta s^{*}\right)\left(\eta+p^{*} / \lambda\right)$; the stable manifold $W^{S}$ is $W^{S}=\left\{(s, i, p, v) \in \mathbb{R}_{4}^{+} \mid\left(s<s^{*}, i<i^{*}, p>p^{*}, v>v^{*}\right) \cup(s>\right.$ $\left.\left.s^{*}, i>i^{*}, p<p^{*}, v<v^{*}\right)\right\}$.

Proof. We choose the Lyapunov function as follows:

$$
V(s, i, p, v)=\frac{1}{2}\left[c_{1} s^{2}+c_{2} i^{2}+c_{3} p^{2}+c_{4} v^{2}\right],
$$

where $c_{i}>0, i=1,2,3,4$, is to be determined suitably. Obviously $V$ is positive definite. The derivative of $V$ along the solution of the equation $\dot{X}(t)=J_{E^{*}} X(t)$, where $X(t)=$ $(s(t), i(t), p(t), v(t))^{T}$, is as follows:

$$
\begin{aligned}
\dot{V}= & c_{1} s \dot{s}+c_{2} \dot{i}+c_{3} p \dot{p}+c_{4} v \dot{v} \\
= & -a c_{1} s^{*} s^{2}+\left(c_{2} v^{*}-a c_{1} s^{*}\right) s i-c_{1} s^{*} s v \\
& -c_{2}\left(\frac{1}{\lambda} p^{*}+\eta\right) i^{2}+\left(c_{3} c p^{*}-\frac{c_{2}}{\lambda} i^{*}\right) i p+\left(c_{2} s^{*}+c_{4} k \eta\right) i v \\
& -c_{3} \epsilon p^{*} p^{2}-c_{4} \mu v^{2} .
\end{aligned}
$$

The symmetric matrix corresponding to $\dot{V}$ is the following:

$$
B=\frac{1}{2}\left[\begin{array}{cccc}
-2 a c_{1} s^{*} & c_{2} v^{*}-a c_{1} s^{*} & 0 & -c_{1} s^{*} \\
c_{2} v^{*}-a c_{1} s^{*} & -2 c_{2}\left(\frac{1}{\lambda} p^{*}+\eta\right) & c_{3} c p^{*}-\frac{c_{2}}{\lambda} i^{*} & c_{2} s^{*}+c_{4} k \eta \\
0 & c_{3} c p^{*}-\frac{c_{2}}{\lambda} i^{*} & -2 c_{3} \epsilon p^{*} & 0 \\
-c_{1} s^{*} & c_{2} s^{*}+c_{4} k \eta & 0 & -2 c_{4} \mu
\end{array}\right] .
$$

The positive equilibrium $E^{*}$ is locally asymptotically stable if $\dot{V}$ is negative definite, which in turn follows if the symmetric matrix $B$ is negative definite; that is, the odd rank principal minor in order is negative and the even rank principal minor in order is positive, which in turn follows if

(i) $-2 a c_{1} s^{*}<0$;

(ii) $4 a c_{1} c_{2}\left((1 / \lambda) p^{*}+\eta\right) s^{*}-\left(c_{2} v^{*}-a c_{1} s^{*}\right)^{2}>0$;

(iii) $-2 a c_{1} s^{*}\left[4 c_{2} c_{3} \epsilon\left(\eta+(1 / \lambda) p^{*}\right) p^{*}-\left(c_{3} p^{*}-\left(c_{2} / \lambda\right) i^{*}\right)^{2}\right]+$ $2 c_{3} \in p^{*}\left(c_{2} v^{*}-a c_{1} s^{*}\right)^{2}<0$

(iv) $-2 c_{4} \mu$ [the left expression of the inequality (iii)] $-4 a c_{1} c_{3} \epsilon p^{*} s^{*}\left(c_{4} k \eta-c_{2} s^{*}\right)^{2}-16 a c_{1} c_{2} c_{3} c_{4} \epsilon k \eta\left(s^{*}\right)^{2} p^{*}>$ 0 .

We choose $c_{2} v^{*}-a c_{1} s^{*}=0, c_{3} p^{*}-\left(c_{2} / \lambda\right) i^{*}=0$, and $c_{4} k \eta-c_{2} s^{*}=0$; then from the above expression, we know if $16 c_{4} \mu a c_{1} s^{*} c_{2} c_{3} \epsilon\left(\eta+(1 / \lambda) p^{*}\right) p^{*}>16 a c_{1} c_{2} c_{3} c_{4} \epsilon \eta\left(s^{*}\right)^{2} p^{*}$, that is, $k<\left(\mu / \eta s^{*}\right)\left(\eta+p^{*} / \lambda\right)$, for the symmetric matrix $B$, the odd rank principal minor in order is negative and the even rank principal minor in order is positive. That is to say, if $k<\left(\mu / \eta s^{*}\right)\left(\eta+p^{*} / \lambda\right)$, the positive equilibrium $E^{*}$ is locally asymptotically stable. Furthermore, to find out the basin of attraction, we choose the Lyapunov function as follows:

$$
\begin{aligned}
V(s, i, p, v)= & s-s^{*}-s^{*} \ln \left(\frac{s}{s^{*}}\right)+\frac{1}{2} c_{1}\left(i-i^{*}\right)^{2} \\
& +\left[p-p^{*}-p^{*} \ln \left(\frac{p}{p^{*}}\right)\right]+\frac{1}{2}\left(v-v^{*}\right)^{2} .
\end{aligned}
$$

Differentiating $V$ along the solution of the model and choosing $c_{1}=a / v^{*}$, we can obtain the stable manifold $W^{S}=$ $\left\{(s, i, p, v) \in \mathbb{R}_{4}^{+} \mid\left(s<s^{*}, i<i^{*}, p>p^{*}, v>v^{*}\right) \cup(s>\right.$ $\left.\left.s^{*}, i>i^{*}, p<p^{*}, v<v^{*}\right)\right\}$. The proof is completed.

Now, we will find out the conditions for which the equilibrium $E^{*}$ enters into Hopf bifurcation. The characteristic equation for $E^{*}$ is

$$
\Lambda^{4}+p_{1} \Lambda^{3}+p_{2} \Lambda^{2}+p_{3} \Lambda+p_{4}=0
$$


where

$$
\begin{aligned}
p_{1}= & a s^{*}+\eta+\frac{1}{\lambda} p^{*}+\epsilon p^{*}+\mu ; \\
p_{2}= & a s^{*}\left(\epsilon p^{*}+\mu\right)+\epsilon p^{*} \mu+\left(\eta+\frac{1}{\lambda} p^{*}\right)\left(a s^{*}+\epsilon p^{*}+\mu\right) \\
& +a s^{*} v^{*}+\frac{c}{\lambda} p^{*} i^{*}-k \eta s^{*} ; \\
p_{3}= & a s^{*} \epsilon p^{*} \mu+\left(\eta+\frac{1}{\lambda} p^{*}\right)\left(\mu \epsilon p^{*}+\epsilon p^{*} a s^{*}+a s^{*} \mu\right) \\
& +\frac{c}{\lambda} p^{*} i^{*}\left(a s^{*}+\mu\right) \\
& +a \epsilon s^{*} p^{*} v^{*}+v^{*} s^{*}(a \mu+k \eta)-k \eta s^{*}\left(a s^{*}+\epsilon p^{*}\right) ; \\
p_{4}= & a \epsilon \mu s^{*} p^{*}\left(\eta+\frac{1}{\lambda} p^{*}\right)+a \mu s^{*} \frac{c}{\lambda} p^{*} i^{*} \\
& +\epsilon s^{*} p^{*} v^{*}(a \mu+k \eta)-a \epsilon \eta k\left(s^{*}\right)^{2} p^{*} .
\end{aligned}
$$

Since $p_{2}, p_{3}$, and $p_{4}$ can be written as $p_{2}=a s^{*}\left(\epsilon p^{*}+\mu\right)+$ $\epsilon p^{*} \mu+\left(\eta+(1 / \lambda) p^{*}\right)\left(a s^{*}+\epsilon p^{*}\right)+\left[\mu\left(\eta+(1 / \lambda) p^{*}\right)-k \eta s^{*}\right]+$ $a s^{*} v^{*}+(c / \lambda) p^{*} i^{*}, p_{3}=a s^{*} \epsilon p^{*} \mu+\left(a s^{*}+\epsilon p^{*}\right)\left[\mu\left(\eta+(1 / \lambda) p^{*}\right)-\right.$ $\left.k \eta s^{*}\right]+\left(\eta+(1 / \lambda) p^{*}\right) \epsilon p^{*} a s^{*}+(c / \lambda) p^{*} i^{*}\left(a s^{*}+\mu\right)+a \epsilon s^{*} p^{*} v^{*}+$ $v^{*} s^{*}(a \mu+k \eta)$, and $p_{4}=a \epsilon s^{*} p^{*}\left[\mu\left(\eta+(1 / \lambda) p^{*}\right)-\eta k s^{*}\right]+$ $a \mu s^{*}(c / \lambda) p^{*} i^{*}+\epsilon s^{*} p^{*} v^{*}(a \mu+k \eta)$, respectively, under the condition $k<\left(\mu / \eta s^{*}\right)\left(\eta+(1 / \lambda) p^{*}\right)$, it is not easy to know $p_{i}>0, i=1,2,3,4$. Let $\Psi(k):(0,+\infty) \rightarrow \mathbb{R}$ be the following continuously differentiable function: $\Psi(k)=$ $p_{1}(k) p_{2}(k) p_{3}(k)-p_{3}^{2}(k)-p_{4}(k) p_{1}^{2}(k)$. A Hopf bifurcation of the equilibrium $E^{*}$ occurs at $k^{*} \in(1,+\infty)$ if and only if (i) $\Psi\left(k^{*}\right)=0,\left.(d \operatorname{Re} \Lambda(k) / d k)\right|_{k^{*}} \neq 0$, where $\Lambda(k)$ is purely imaginary at $k=k^{*}$, and (ii) all other eigenvalues are of negative real parts.

The existence of $k^{*}$ can be obtained by solving $\Psi(k)=0$. At $k=k^{*}, p_{1} p_{2} p_{3}=p_{3}^{2}+p_{4} p_{1}^{2}$; that is, $p_{2}=p_{3} / p_{1}+p_{4} p_{1} / p_{3}$, which deduces that the characteristic equation can be written as $\left(\Lambda^{2}+p_{3} / p_{1}\right)\left(\Lambda^{2}+p_{1} \Lambda+p_{1} p_{4} / p_{3}\right)=0$. So there exist a pair of purely imaginary $\Lambda_{1}, \Lambda_{2}= \pm \sqrt{p_{3} / p_{1}}$, respectively; other eigenvalues $\Lambda_{3}, \Lambda_{4}$ satisfy $\Lambda_{3}+\Lambda_{4}=-p_{1}, \Lambda_{3} \Lambda_{4}=p_{1} p_{4} / p_{3}$. Denote $\omega_{0}=\operatorname{Im} \Lambda_{1}\left(k^{*}\right)=\sqrt{p_{3} / p_{1}}$; then $\omega_{0}^{2}+\Lambda_{3} \Lambda_{4}=$ $\left(p_{3}^{2}+p_{1}^{2} p_{4}\right) / p_{1} p_{3}=p_{2}$ since $\Psi\left(k^{*}\right)=0, \omega_{0}^{2}\left(\Lambda_{3}+\Lambda_{4}\right)=-p_{3}$, and $\omega_{0}^{2} \Lambda_{3} \Lambda_{4}=p_{4}$. If $\Lambda_{3}$ and $\Lambda_{4}$ are complex conjugates, then from $\Lambda_{3}+\Lambda_{4}=-p_{1}$ we know $\operatorname{Re} \Lambda_{3}=-p_{1} / 2<0$; if $\Lambda_{3}$ and $\Lambda_{4}$ are real roots, then $\Lambda_{3} \Lambda_{4}>0$ based on the fact that $\omega_{0}^{2} \Lambda_{3} \Lambda_{4}=p_{4}>0$; furthermore, since $\Lambda_{3}+\Lambda_{4}=$ $-p_{1}<0$, then $\Lambda_{3}<0, \Lambda_{4}<0$. In summary, for the characteristic equation, besides $\Lambda_{1}$ and $\Lambda_{2}$, other eigenvalues are negative real parts. Now we show $\left.(d \operatorname{Re} \Lambda(k) / d k)\right|_{k^{*}} \neq 0$. In fact, let $\Lambda(k)=\beta_{1}(k)+i \beta_{2}(k)$; substituting it into the characteristic equation for the positive equilibrium $E^{*}$ and calculating the derivative on $k$, the following expression can be given through some calculations and arrangement:

$$
\begin{aligned}
& {\left[A(k) \beta_{1}^{\prime}-L(k) \beta_{2}^{\prime}+M(k)\right]} \\
& \quad+\left[L(k) \beta_{1}^{\prime}+A(k) \beta_{2}^{\prime}+N(k)\right] i=0,
\end{aligned}
$$

where

$$
\begin{gathered}
A(k)=4 \beta_{1}^{3}-12 \beta_{1} \beta_{2}^{2}+3 p_{1} \beta_{1}^{2}-3 p_{1} \beta_{2}^{2}+2 p_{2} \beta_{1}+p_{3}, \\
L(k)=12 \beta_{1}^{2} \beta_{2}+6 p_{1} \beta_{1} \beta_{2}-4 \beta_{2}^{3}+2 p_{2} \beta_{2}, \\
M(k)=p_{1}^{\prime} \beta_{1}^{3}-3 p_{1}^{\prime} \beta_{1} \beta_{2}^{2}+p_{2}^{\prime} \beta_{1}^{2}-p_{2}^{\prime} \beta_{2}^{2}+p_{3}^{\prime} \beta_{1}+p_{4}^{\prime}, \\
N(k)=-p_{1}^{\prime} \beta_{2}^{3}+3 p_{1}^{\prime} \beta_{1}^{2} \beta_{2}+2 p_{2}^{\prime} \beta_{1} \beta_{2}+p_{3}^{\prime} \beta_{2} .
\end{gathered}
$$

It deduces that

$$
\begin{aligned}
& A(k) \beta_{1}^{\prime}-L(k) \beta_{2}^{\prime}+M(k)=0, \\
& L(k) \beta_{1}^{\prime}+A(k) \beta_{2}^{\prime}+N(k)=0 .
\end{aligned}
$$

Solving the above equations on $\beta_{1}^{\prime}, \beta_{2}^{\prime}$, we have

$$
\left.\frac{d \operatorname{Re} \Lambda(k)}{d k}\right|_{k^{*}}=\frac{L\left(k^{*}\right) N\left(k^{*}\right)+A\left(k^{*}\right) M\left(k^{*}\right)}{A^{2}\left(k^{*}\right)+L^{2}\left(k^{*}\right)} \neq 0 .
$$

At this point, the conditions of Hopf bifurcation for positive equilibrium $E^{*}$ are verified. In summary, we have the following theorem.

Theorem 3. There exists $a k^{*} \in(\mu,+\infty)$; the Hopf bifurcation for the positive equilibrium $E^{*}$ occurs at $k=k^{*}$. The period of the bifurcating periodic orbits close to $k=k^{*}$ is $2 \pi / \omega_{0}$, where $\omega_{0}=\sqrt{p_{3}\left(k^{*}\right) / p_{1}\left(k^{*}\right)}$.

In the next section we will consider the stability of bifurcating periodic orbits applying Poore's condition [19].

\section{Stability of the Bifurcating Periodic Solution}

We apply Poore's condition for verification of the orbital stability of the Hopf bifurcating limit cycle. For convenience, we list the preliminaries firstly. Let a real, $n$-dimensional $(n \geq$ 2 ), first-order system of autonomous differential equations be of the form

$$
\frac{d x}{d t}=\widehat{F}(x, v),
$$

where $v=\left(v_{1}, v_{2}, \ldots, v_{m}\right)^{T}$ denotes a vector of $m$-real parameters. There must firstly exist a combination of the parameters, say, $v_{0}$; a critical point $a^{0}$, such that the variational matrix $\widehat{F}_{x}\left(a^{0}, v^{0}\right)$ has exactly two, nonzero, purely imaginary eigenvalues, say, $\pm i \omega_{0}$ with $\omega_{0}>0$; and other $n-2$ eigenvalues with nonzero real parts. To vary one, some, or all of the $m$ parameters, an $m$-dimensional vector function $f(\varepsilon)$ is introduced with the property that $f(0)=0$, and hence we confine our analysis to the following system of ODE:

$$
\frac{d x}{d t}=F(x, \varepsilon):=\widehat{F}\left(x, \nu^{0}+\varepsilon\right) .
$$

It follows from the definition of $F(x, \varepsilon)$ that $F\left(a^{0}, 0\right)=0$ and eigenvalues of $F_{x}\left(a^{0}, 0\right)=0$ are the same as those of $\widehat{F}\left(a^{0}, \nu^{0}\right)$. 
It is assumed that $F(x, \varepsilon) \in C^{k}\left[D \times\left(-\varepsilon_{0}, \varepsilon_{0}\right)\right]$, where $k \geq 3$ and $D$ is a domain in $\mathbb{R}^{n}$ containing $a^{0}$ and $\varepsilon_{0}>0$. As there is no nonzero eigenvalue of the variational matrix, $F_{x}\left(a^{0}, 0\right) \neq 0 \in$ $\mathbb{R}^{n}$, and hence the implicit function theorem guarantees the existence of a critical point $a^{\varepsilon}$ which is $k$-times continuously differentiable in $\varepsilon$ and satisfies $F\left(a^{\varepsilon}, \varepsilon\right)=0$ for $\varepsilon$ in a small neighborhood of $\varepsilon=0$. Using this definition of $a^{\varepsilon}$, a change of variables is introduced:

$$
\begin{gathered}
x=a^{\varepsilon}+\mu^{\prime} y, \quad\left(1+\mu^{\prime} \eta\right)=t, \\
\varepsilon=\mu^{\prime} \delta .
\end{gathered}
$$

This reduces the differential (25) to the following form:

$$
\frac{d y}{d t}=A^{0} y+\mu^{\prime} G\left(y, \mu^{\prime}, \delta, \eta\right)
$$

where $A^{0}=F_{x}\left(a^{0}, 0\right)$ and $G$ is defined as given in [19]. Thus, the problem of periodic solutions of (25) is reduced to a perturbation problem in the small parameter of $\mu^{\prime}$.

Now, the stability information of the bifurcating periodic orbits is contained in the following theorem of Poore, coupled with an algebraic expression, which completely reduces the determination of stability to an algebraic problem. By the assumptions of Theorem 2.3 [19], the differential equation in (27) is continuously differentiable in $\mu^{\prime}$, where $\delta=\delta\left(\mu^{\prime}\right)$ and $\eta=\eta\left(\mu^{\prime}\right)$, and in the function $y$ in a neighborhood of the periodic orbit. Thus, the existing periodic orbit will be asymptotically orbitally stable with asymptotic phase if $n-1$ of the characteristic multipliers of the variational equation have moduli less than one. The following theorem developed by Poore about the modulus of each of the characteristic multipliers reduces the condition of orbital stability of the Hopf bifurcating limit cycle to an algebraic expression as follows.

Theorem 4. Let $F(x, \varepsilon)$ satisfy the hypotheses in Theorem 2.3 [19] and let $u$ and $v$ denote the left and right eigenvectors, respectively, for the eigenvalues $i \omega_{0}\left(\omega_{0}>0\right)$ of the variational matrix $A^{0}$. If $u$ and $v$ are normalized in the sense that $u v=1$, then

$$
\begin{aligned}
8 \alpha^{\prime}(0) \delta^{\prime}(0)+i 8\left(\omega^{\prime}(0) \delta^{\prime}(0)+\omega_{0} \eta^{\prime}(0)\right) & \\
=b(0) \cdot b(0)\{ & -u F_{x x x} v v \bar{v}+2 u F_{x x} v A^{0-1} F_{x x} v \bar{v} \\
& \left.+u F_{x x} \bar{v}\left(A^{0}-2 i \omega\right)^{-1} F_{x x} v \bar{v}\right\}
\end{aligned}
$$

where $A^{0}=F_{x}\left(a^{0}, 0\right), F_{x x}=F_{x x}\left(a^{0}, 0\right), F_{x x x}=F_{x x x}\left(a^{0}, 0\right)$, $b(0)$ is the $\mu^{\prime}=0$ value of the two-dimensional vector $b\left(\mu^{\prime}\right)$, which occurs in $\bigcup y\left(s, \mu^{\prime}\right)=\Phi(s) b\left(\mu^{\prime}\right)$, and $\bar{v}$ denotes the complex conjugate of $v$.
Written out in component form, the above expression reduces to

$$
\begin{aligned}
8 \alpha^{\prime}(0) \delta^{\prime}(0)+i 8\left(\omega^{\prime}(0) \delta^{\prime}(0)+\omega_{0} \eta^{\prime}(0)\right) & \\
=b(0) \cdot b(0)\{- & u_{l} F_{x_{j} x_{k} x_{p}}^{l} v_{j} v_{k} \bar{v}_{p} \\
& +2 u_{l} F_{x_{j} x_{k}}^{l} v_{j}\left(A^{0-1}\right)_{k r} F_{x_{p} x_{q}}^{r} v_{p} \bar{v}_{q} \\
& \left.+u_{l} F_{x_{j} x_{k}}^{l} \bar{v}_{j}\left(\left(A^{0}-2 i \omega\right)^{-1}\right)_{k r} F_{x_{p} x_{q}}^{r} v_{p} \bar{v}_{q}\right\},
\end{aligned}
$$

where the repeated indices within each term imply a sum from 1 to $n$ and all the derivatives of $F$ are evaluated at the equilibrium $a^{0}$. The sign of the real and imaginary parts of the right-hand side of expression in Theorem 4 is independent of the choice of $b\left(\mu^{\prime}\right)$ in $\bigcup y\left(s, \mu^{\prime}\right)=\Phi(s) b\left(\mu^{\prime}\right)$ and the eigenvectors $u$ and $v$ so long as $b(0) \cdot b(0)>0$ and $u v=$ 1. So positivity of the real part of the above expression in parenthesis really indicates the orbital stability of the periodic solution arising out of Hopf bifurcation.

For model (7) we considered, let the right four equations be $F^{1}=a s(1-s-i)-v s, F^{2}=v s-(1 / \lambda) i p-\eta i, F^{3}=$ $p(-d-\epsilon p+c i)$, and $F^{4}=-\mu v+k \eta i$, respectively. To verify that the conditions of Theorem 4 are satisfied, we will calculate the second- and the third-order derivatives of $F^{l}, l=1,2,3,4$, as follows:

$$
\begin{gathered}
F_{s s}^{1}=-2 a, \quad F_{s i}^{1}=F_{i s}^{1}=-a, \quad F_{s v}^{1}=F_{v s}^{1}=-1 ; \\
F_{s v}^{2}=F_{v s}^{2}=1, \quad F_{i p}^{2}=F_{p i}^{2}=-\frac{1}{\lambda} ; \\
F_{p p}^{3}=-2 \epsilon, \quad F_{i p}^{3}=F_{p i}^{3}=c .
\end{gathered}
$$

Other second-order and third-order derivatives are all zero. The inverse matrix $Q$ of $J_{E^{*}}-2 i \omega_{0}$ and the left and right eigenvectors $u$ and $v$, respectively, in the sense that $u v=1$, of the variational matrix $J_{E^{*}}$ can be calculated. Based on the preliminary work, we can calculate expression (29) in parenthesis as follows:

$$
\begin{gathered}
-u_{l} F_{x_{j} x_{k} x_{p}}^{l} v_{j} v_{k} \bar{v}_{p}=0, \\
2 u_{l} F_{x_{j} x_{k}}^{l} v_{j}\left(A^{0-1}\right)_{k r} F_{x_{p} x_{q}}^{r} v_{p} \bar{v}_{q} \\
=2\left[u_{1}\left(F_{s s}^{1} v_{1}+F_{i s}^{1} v_{2}+F_{v s}^{1} v_{4}\right)+u_{2} F_{v s}^{2} v_{4}\right] \\
\times\left[Q_{11} I+Q_{12} I I+Q_{13} I I I\right] \\
+2\left[u_{1} F_{s i}^{1} v_{1}+u_{2} F_{p i}^{2} v_{3}+u_{3} F_{p i}^{3} v_{3}\right] \\
\times\left[Q_{21} I+Q_{22} I I+Q_{23} I I I\right] \\
+2\left[u_{2} F_{i p}^{2} v_{2}+u_{3}\left(F_{p p}^{3} v_{3}+F_{i p}^{3} v_{2}\right)\right] \\
\times\left[Q_{31} I+Q_{32} I I+Q_{33} I I I\right] \\
+2\left(u_{1} F_{s v}^{1} v_{1}+u_{2} F_{s v}^{2} v_{1}\right)\left[Q_{41} I+Q_{42} I I+Q_{43} I I I\right]
\end{gathered}
$$




$$
\begin{aligned}
u_{l} F_{x_{j} x_{k}}^{l} \bar{v}_{j} & \left(\left(A^{0}-2 i \omega\right)^{-1}\right)_{k r} F_{x_{p} x_{q}}^{r} v_{p} \bar{v}_{q} \\
= & {\left[u_{1}\left(F_{s s}^{1} \bar{v}_{1}+F_{i s}^{1} \bar{v}_{2}+F_{v s}^{1} \bar{v}_{4}\right)+u_{2} F_{v s}^{2} \bar{v}_{4}\right] } \\
& \times\left[\left(Q_{\omega_{0}}\right)_{11} I+\left(Q_{\omega_{0}}\right)_{12} I I+\left(Q_{\omega_{0}}\right)_{13} I I I\right] \\
& +\left(u_{1} F_{s i}^{1} \bar{v}_{1}+u_{2} F_{p i}^{2} \bar{v}_{3}+u_{3} F_{p i}^{3} \bar{v}_{3}\right) \\
& \times\left[\left(Q_{\omega_{0}}\right)_{21} I+\left(Q_{\omega_{0}}\right)_{22} I I+\left(Q_{\omega_{0}}\right)_{23} I I I\right] \\
& +\left[u_{2} F_{i p}^{2} \bar{v}_{2}+u_{3}\left(F_{p p}^{3} \bar{v}_{3}+F_{i p}^{3} \bar{v}_{2}\right)\right] \\
& \times\left[\left(Q_{\omega_{0}}\right)_{31} I+\left(Q_{\omega_{0}}\right)_{32} I I+\left(Q_{\omega_{0}}\right)_{33} I I I\right] \\
& +\left[u_{1} F_{s v}^{1} \bar{v}_{1}+u_{2} F_{s v}^{2} \bar{v}_{1}\right] \\
& \times\left[\left(Q_{\omega_{0}}\right)_{41} I+\left(Q_{\omega_{0}}\right)_{42} I I+\left(Q_{\omega_{0}}\right)_{43} I I I\right]
\end{aligned}
$$

where

$$
\begin{gathered}
I=F_{s s}^{1}\left|v_{1}\right|^{2}+F_{s i}^{1}\left(v_{1} \bar{v}_{2}+v_{2} \bar{v}_{1}\right)+F_{s v}^{1}\left(v_{1} \bar{v}_{4}+v_{4} \bar{v}_{1}\right), \\
I I=F_{s v}^{2}\left(v_{1} \bar{v}_{4}+v_{4} \bar{v}_{1}\right)+F_{i p}^{2}\left(v_{3} \bar{v}_{2}+v_{2} \bar{v}_{3}\right), \\
I I I=F_{i p}^{3}\left(v_{2} \bar{v}_{3}+v_{3} \bar{v}_{2}\right)+F_{p p}^{3}\left|v_{3}\right|^{2} .
\end{gathered}
$$

Putting the values of $F_{x x x}, F_{x x}, u$, and $v$ and components of the matrix $Q$ in terms of the parameters of the model, the positivity of the real part of expression (29) in parenthesis can be deduced. This in turn indicates the orbital stability of the limit cycle arising out of Hopf bifurcation.

\section{Extinction and Permanence with Impulsive Effect}

In the sections ahead, we focus our attention on model (6) or (7), in which the growth of the predator and the virus is continuous. But in fact, it is needed to throw in the predator or virus according to the pest control at some moments for better effects. So in this section, we develop model (6) into an impulsive system as follows and the presence of impulses gives the system a mixed nature, both continuous and discrete:

$$
\begin{gathered}
\frac{d S}{d t}=r S\left(1-\frac{S+I}{K}\right)-\lambda S V, \\
\frac{d I}{d t}=\lambda S V-l I P-\xi I, \\
\frac{d P}{d t}=P\left(-d-\epsilon_{P} P+C l I\right), \\
\frac{d V}{d t}=-\mu_{v} V+k \xi I, \quad t \neq n \tau,
\end{gathered}
$$

$$
\begin{gathered}
S\left(n \tau^{+}\right)=S\left(n \tau^{-}\right), \\
I\left(n \tau^{+}\right)=I\left(n \tau^{-}\right), \\
P\left(n \tau^{+}\right)=P\left(n \tau^{-}\right)+b, \\
V\left(n \tau^{+}\right)=V\left(n \tau^{-}\right)+q, \quad t=n \tau,
\end{gathered}
$$

where the predator and virus are thrown at $n \tau, n=0,1,2, \ldots$, $\tau$ is the period, and $b, q$ are the amount of impulsive stocking on the predator and the virus; $b, q$ are positive constants. Other parameters are the same as model (6). $S\left(n \tau^{+}\right), I\left(n \tau^{+}\right)$, $P\left(n \tau^{+}\right)$, and $V\left(n \tau^{+}\right)$represent the densities of the susceptible pest, the infected pest by virus, the predator, and the virus, respectively: $S\left(n \tau^{+}\right)=\lim _{t \rightarrow n \tau^{+}} S(t), I\left(n \tau^{+}\right)=\lim _{t \rightarrow n \tau^{+}} I(t)$, $P\left(n \tau^{+}\right)=\lim _{t \rightarrow n \tau^{+}} P(t)$, and $V\left(n \tau^{+}\right)=\lim _{t \rightarrow n \tau^{+}} V(t) ; S(t)$, $I(t), P(t)$, and $V(t)$ are left continuous at $n \tau$; that is, $S(n \tau)=$ $\lim _{t \rightarrow n \tau^{-}} S(t), I(n \tau)=\lim _{t \rightarrow n \tau^{-}} I(t), P(n \tau)=\lim _{t \rightarrow n \tau^{-}} P(t)$, and $V(n \tau)=\lim _{t \rightarrow n \tau^{-}} V(t)$. In this section, we mainly consider the global stability of pest-eradication solution and the uniform permanence of system (33); before introducing our main results, we give some preliminaries needed next.

For the system

$$
\begin{gathered}
\frac{d V}{d t}=-\mu_{v} V, \quad t \neq n \tau, \\
V\left(n \tau^{+}\right)=V\left(n \tau^{-}\right)+q, \quad t=n \tau, \\
V\left(0^{+}\right)=V_{0} \geq 0,
\end{gathered}
$$

we have the following lemma.

Lemma 5. System (34) has a unique positive periodic solution $V^{*}(t)$ with period $\tau$ if and only if the initial value $V^{*}\left(0^{+}\right)=$ $q /\left(1-e^{-\mu_{v} \tau}\right)$, where $V^{*}(t)=q e^{-\mu_{v}(t-n \tau)} /\left(1-e^{-\mu_{v} \tau}\right), n \tau<t \leq$ $(n+1) \tau$, and $V^{*}(t)$ is globally asymptotically stable; in addition, every solution $V(t)$ of system (34) can be written as $V(t)=$ $V^{*}(t)+\left(V\left(0^{+}\right)-V^{*}\left(0^{+}\right)\right) e^{-\mu_{v} t}, n \tau<t \leq(n+1) \tau$.

For the system

$$
\begin{gathered}
\frac{d P}{d t}=P\left(-d-\epsilon_{P} P\right), \quad t \neq n \tau, \\
P\left(n \tau^{+}\right)=P\left(n \tau^{-}\right)+b, \quad t=n \tau, \\
P\left(0^{+}\right)=P_{0} \geq 0,
\end{gathered}
$$

we have the following lemma.

Lemma 6. System (35) has a unique positive periodic solution $P^{*}(t)$ with period $\tau$ if and only if the initial value $P^{*}\left(0^{+}\right)=$ $-\left[\left(1-e^{d \tau}\right)\left(d / \epsilon_{p}-b\right)+\sqrt{\Delta}\right] /\left[2\left(1-e^{d \tau}\right)\right]$, where $\Delta=(1-$ $\left.e^{d \tau}\right)^{2}\left(d / \epsilon_{p}-b\right)^{2}-\left(4 d / \epsilon_{p}\right)\left(1-e^{d \tau}\right) b e^{d \tau}, P^{*}(t)=\left(-d / \epsilon_{p}\right) /(1-$ $\left.\left(d / P^{*} \epsilon_{p}+1\right) e^{d(t-n \tau)}\right), n \tau<t \leq(n+1) \tau$, and $P^{*}(t)$ is globally asymptotically stable. 
Lemma 7. There exists a constant $L>0$ such that $S(t), I(t)$, $P(t)$, and $V(t) \leq L$ for each solution of (33) with $t$ being large enough.

The solution of system (33) corresponding to $I=0$ is called pest-eradication solution $\left(0,0, P^{*}(t), V^{*}(t)\right)$ whose explicit form can be obtained by Lemmas 5 and 6 , where $P^{*}(t)=\left(-d / \epsilon_{p}\right) /\left(1-\left(d / P^{*} \epsilon_{p}+1\right) e^{d(t-n \tau)}\right)$ and $V^{*}(t)=$ $q e^{-\mu_{v}(t-n \tau)} /\left(1-e^{-\mu_{v} \tau}\right), n \tau<t \leq(n+1) \tau$, with initial values $P^{*}\left(0^{+}\right), V^{*}\left(0^{+}\right)$as Lemmas 5 and 6 , respectively. Now we consider the stability of pest-eradication solution. The Jacobi matrix $\mathbf{A}(\mathbf{t})$ at $\left(0,0, P^{*}(t), V^{*}(t)\right)$ is

$$
\mathbf{A}(\mathbf{t})=\left[\begin{array}{cccc}
r-\lambda V^{*}(t) & 0 & 0 & 0 \\
\lambda V^{*}(t) & -l P^{*}(t)-\xi & 0 & 0 \\
0 & C l P^{*}(t) & -d-2 \epsilon_{p} P^{*}(t) & 0 \\
0 & k \xi & 0 & -\mu_{v}
\end{array}\right]
$$

we can calculate the monodromy matrix $\mathbf{M}(\mathbf{T})=\mathbf{B} e^{\int_{0}^{T} A(t) d t}$, where $\mathbf{B}$ is identity matrix and $\mathbf{M}(\mathbf{T})$ is just as follows:

$$
\left[\begin{array}{cccc}
\exp \left(r \tau-\lambda \int_{0}^{\tau} V^{*} d t\right) & 0 & 0 & 0 \\
\Delta & \exp \left(-l \int_{0}^{\tau} P^{*} d t-\xi \tau\right) & 0 & 0 \\
\Delta & \Delta & \exp \left(-d \tau-2 \epsilon_{p} \int_{0}^{\tau} P^{*} d t\right) & 0 \\
\Delta & \Delta & \Delta & e^{-\mu_{v} \tau}
\end{array}\right]
$$

there is no need to calculate the exact forms of $\Delta$ since they are not required for the following analyses. The Floquet multipliers are $\mu_{1}=\exp \left(r \tau-\lambda \int_{0}^{\tau} V^{*} d t\right), \mu_{2}=$ $\exp \left(-l \int_{0}^{\tau} P^{*} d t-\xi \tau\right), \mu_{3}=\exp \left(-d \tau-2 \epsilon_{p} \int_{0}^{\tau} P^{*} d t\right)$, and $\mu_{4}=e^{-\mu_{v} \tau}$, respectively. Obviously, $0<\mu_{2}, \mu_{3}, \mu_{4}<1$, so the stability of $\left(0,0, P^{*}(t), V^{*}(t)\right)$ is decided by whether $\mu_{1}=\exp \left(r \tau-\lambda \int_{0}^{\tau} V^{*} d t\right)<1$; that is, when $\tau<\lambda q / r \mu_{v}$, the pest-eradication solution is locally stable; further we can prove that it is globally asymptotically stable.

Theorem 8. If $\tau<\lambda q / r \mu_{v}$, then the pest-eradication solution $\left(0,0, P^{*}(t), V^{*}(t)\right)$ of system (33) is globally asymptotically stable.

The pest-eradication solution $\left(0,0, P^{*}(t), V^{*}(t)\right)$ is globally asymptotically stable when $\tau<\lambda q / r \mu_{v}$; that is to say, the pest population is eradicated totally. But in practice, we only need to control the pest population under the economic threshold level (ETL), instead of eradicating it totally, and hope the pest population, the predator (natural enemy), and the virus population can coexist when the pests do not bring about immense economic losses. As for this, we have the uniform permanence of system (33); see the next theorem.

Theorem 9. If $\tau>\lambda q / r \mu_{v}$, then system (33) is uniformly permanent.

The proofs of the lemmas and the theorems listed in this section are all omitted here since they are similar to Hui and Zhu [21].

\section{Numerical Simulations}

In this section, we use numerical simulations to illustrate the dynamical behaviors of system (7) and (33). We choose $k$ as bifurcation parameter; for system (7), the other parameter values are taken as $a=0.1, \lambda=15, \eta=0.06, d=0.1$, $\epsilon=0.05, c=0.5$, and $\mu=0.6$; for system (33), the other parameter values are taken as $r=20, K=10000, \lambda=0.001$, $l=0.01, \xi=10, d=3.1, \epsilon_{p}=0.005, C=0.5, \mu_{v}=10, b=1$, $q=2$, and $\tau=1$.

Firstly, Figure 1 illustrates that the disease-free equilibrium $E_{1}$ is locally asymptotically stable when $k \leq \mu$. It is calculated that $d_{i}(\bar{s})>0(i=1,2,3)$ and $H(\bar{s})>0$ with $k \in$ $(0.6,8.865496710)$. Therefore, the disease-free equilibrium $E_{1}$ is unstable and $E_{2}$ is locally asymptotically stable when $k \in$ $(0.6,8.865496710)$ (see Figure 2). A Hopf bifurcation of the predator-free equilibrium $E_{2}$ occurs at $k=8.865496710$ due to $H(\bar{s})=0$ and $\left.(d H / d \bar{s})\right|_{\bar{s}_{0}} \neq 0$. In this case, there is a periodic solution for system (7) (see Figure 3). When $k \in(1,3)$, the positive equilibrium $E^{*}$ exists and it is locally asymptotically stable. Figure 4 illustrates the stability of the positive equilibrium $E^{*}$.

Figure 5 is the bifurcation graph of system (7). The virus replication factor $k$ is chosen as the bifurcation parameter. When $1<k<8.866$, there is a stable positive equilibrium $E^{*}$; when $k>8.866$, the positive equilibrium $E^{*}$ is unstable and the Hopf bifurcation appears at $k=8.86$; the stable periodic solution appears. Figure 6 illustrates the bifurcation phenomenon of the impulsive system (33). When $1<k<14$, there exists a stable periodic solution; when $k>14$, the system (33) enters into the chaos state.

\section{Conclusions}

In this paper, from Sections 1 to 5 we focus our attention on an epidemic-species hybrid system without impulse influence. From our analyses, we found the virus replication factor $k$ plays a very important role for dynamics of system. The system always has two equilibria, namely, the vanishing 


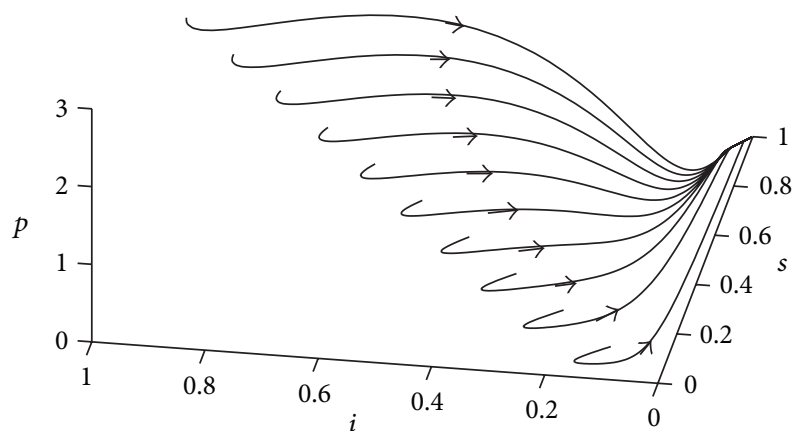

FIGURE 1: Locally asymptotical stability of the disease-free equilibrium $E_{1}$ when $k \leq \mu$, where $k=0.6$.

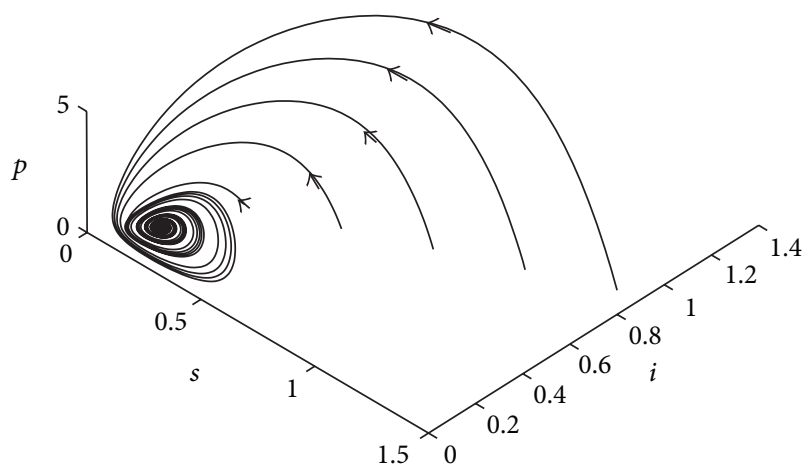

FIGURE 2: Locally asymptotical stability of the predator-free equilibrium $E_{2}$ when $k>\mu$ and $H(\bar{s})>0$, where $k=4$.

equilibrium $E_{0}(0,0,0,0)$ and the disease-free equilibrium $E_{1}(1,0,0,0)$. When the virus replication factor $k$ is larger than its natural death rate $\mu$, that is, $k>\mu$, the predatorfree equilibrium appears; furthermore, the virus replication factor $k$ satisfies $k>\mu$ and $d / c<(\eta a \mu \lambda \epsilon(k-\mu)+$ $\left.a d \mu^{2}\right) /\left(k \eta a \mu \lambda \epsilon+k^{2} \eta^{2} \lambda \epsilon+a c \mu^{2}\right)<a \mu /(a \mu+k \eta)$; the positive equilibrium $E^{*}$ exists. The vanishing equilibrium $E_{0}(0,0,0,0)$ is a saddle point with three-dimensional stable and onedimensional unstable manifold. Biologically, it means that in the absence of prey, that is, the insect pest, the predator and virus population becomes extinct. When $1<k<\mu$, the disease-free equilibrium $E_{1}$ is locally asymptotically stable; it means that if the virus replication parameter or virulence of the applied virus on the system is less than its natural death rate $\mu$, then only $k$ viruses obtained from lysis of the infected pest are not enough to maintain the viral infection in the pest population. When $k=\mu$, the system passes through saddle-node bifurcation and for value of $k$ higher than $\mu$, the system gives two branches of equilibria, namely, the diseasefree equilibrium $E_{1}$ and the predator-free equilibrium $E_{2}$, one hyperbolic equilibrium with three-dimensional stable manifold and one nonhyperbolic equilibrium, respectively; that is, for $k>\mu, E_{1}$ becomes unstable and $E_{2}$ becomes feasible. Biologically, for spreading of infection into the system, the virus replication factor $k$ must be greater than $\mu$. When $k>\mu$, the predator-free equilibrium $E_{2}$ is feasible and stable. For higher value of $k$, there exists a $\bar{s}_{0}$ which

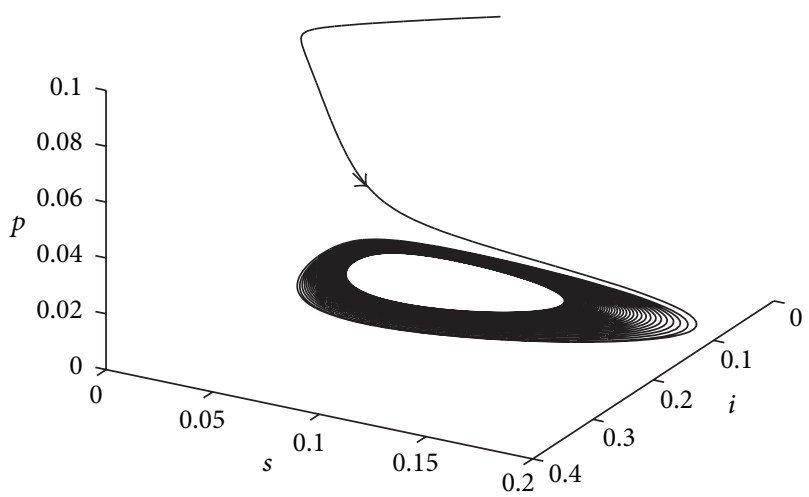

Figure 3: A Hopf bifurcation of the predator-free equilibrium $E_{2}$ occurs when $k>\mu$ and $H(\bar{s})=0,\left.(d H / d \bar{s})\right|_{\bar{s}_{0}} \neq 0$, where $k=9$.

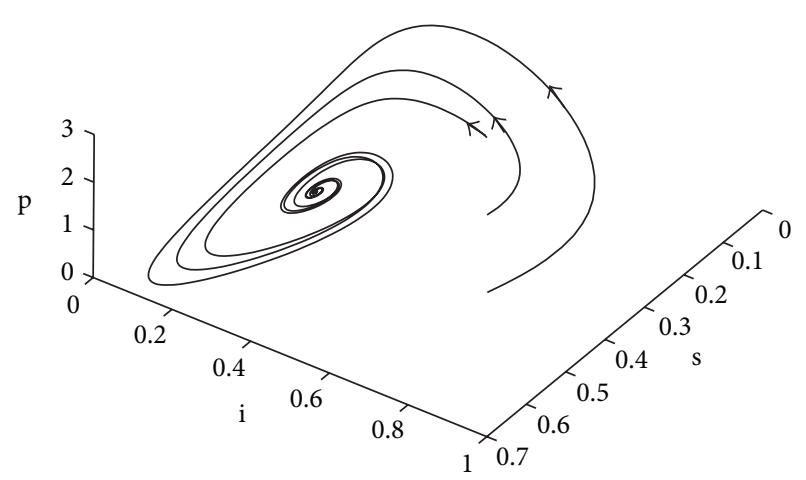

FIGURE 4: Stability of the positive equilibrium $E^{*}$ when $k<\mu(\eta+$ $\left.p^{*} / \lambda\right) /\left(\eta s^{*}\right)$, where $k=2$.

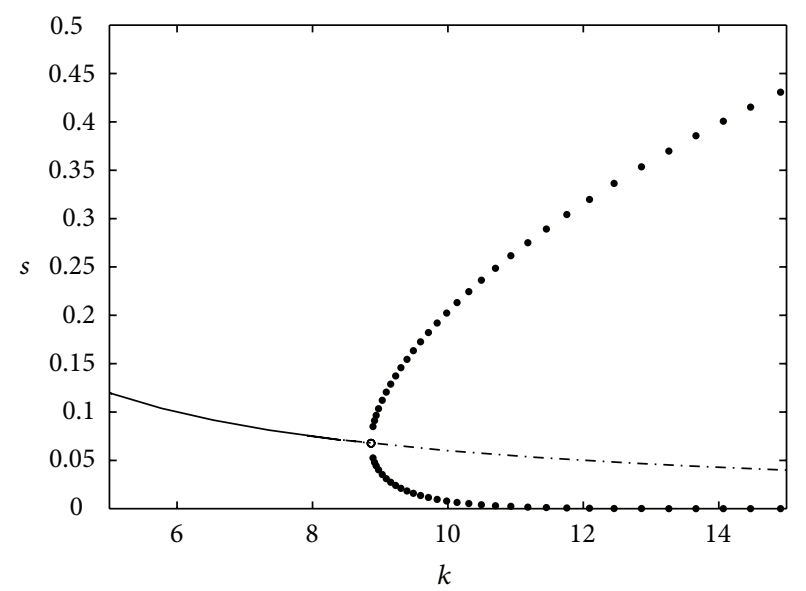

FIGURE 5: Bifurcation of the system (7): bifurcation parameter is chosen as the virus replication factor $k$, where other parameters are $a=0.1, \lambda=15, \eta=0.06, d=0.1, \epsilon=0.05, c=0.5$, and $\mu=0.6$. Horizontal axis represents the bifurcation parameter $k$ and vertical axis represents the positive equilibrium state of the susceptible pest; the full line represents stable equilibrium state and the dotted line represents unstable equilibrium state; the small dots represent the maximum and the minimum amplitude of limit cycle bifurcating from the positive equilibrium and the Hopf bifurcation appears at $k=8.86$. 


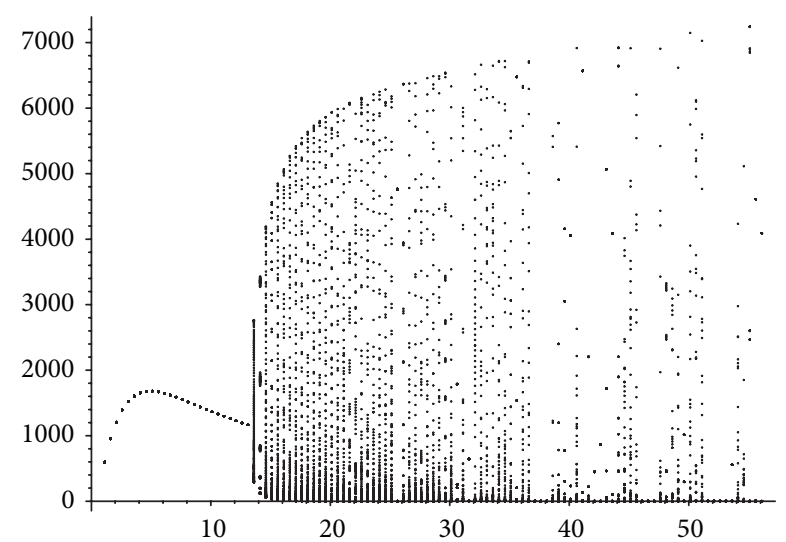

FIGURE 6: Bifurcation of the impulsive system (33): bifurcation parameter is chosen as the virus replication factor $k$, where other parameters are $r=20, K=10000, \lambda=0.001, l=0.01, \xi=10$, $d=3.1, \epsilon_{p}=0.005, C=0.5, \mu_{v}=10, b=1, q=2$, and $\tau=1$. Horizontal axis represents the bifurcation parameter $k$ and vertical axis represents the densities of the infected pest.

deduces that there exists a $k_{0}$ such that at $k=k_{0}$ the system enters into a Hopf bifurcation. When $k>k_{0}$ the solution becomes unstable and gives a periodic orbit of small amplitude near $k_{0}$. For the positive equilibrium $E^{*}$, there exists a $k^{*} \in(\mu,+\infty)$, the positive equilibrium is locally asymptotically stable if $\mu<k<k^{*}$, and the Hopf bifurcation occurs at $k=k^{*}$, bifurcating a periodic orbit which is orbitally stable applying Poore's condition for verification. Considering, in practice, that the predator and the virus are thrown at some moments, lastly in Section 6, we think about the corresponding impulsive equation to model (6). The results have shown that when the impulsive releasing period $\tau<\lambda q / r \mu_{v}$, then the pest-eradication solution $\left(0,0, P^{*}(t), V^{*}(t)\right)$ of system is globally asymptotically stable; otherwise the system is uniformly permanent. So the pest management can be realized by controlling the impulsive releasing period.

\section{Conflict of Interests}

The authors declare that there is no conflict of interests regarding the publication of this paper.

\section{Acknowledgments}

The authors would like to thank the referee, Professor H. F. Huo, for his careful reading of the original paper and giving meaningful suggestions to improve the quality of the paper. At the same time, they thank Professor S. Y. Tang for his instructive advice and useful suggestions for the numerical simulation. This research was supported by Guangxi Natural Science Foundation 2012GXNSFAA053011 and Foundation of Guangxi Educational Committee (no. YB2014203).

\section{References}

[1] B. S. Goh, Management and Analysis of Biological Populations, Elsevier Science, Amsterdam, The Netherlands, 1980.

[2] L. A. Lacey and M. S. Goettel, "Current developments in microbial control of insect pests and prospects for the early $21 \mathrm{st}$ century," Entomophaga, vol. 40, no. 1, pp. 3-27, 1995.

[3] D. R. Dent, "Integrated pest management and microbial insecticides," in Microbial Insecticides: Novelty or Necessity? H. F. Evans, Ed., Proceedings of the British Crop Protection Council Symposium, 68, pp. 127-138, 1997.

[4] R. Georgis, "Commercial prospects of microbial insecticides in agriculture," in Microbial Insecticides: Novelty or Necessity? H. F. Evans, Ed., vol. 68 of Proceedings of the British Crop Protection Council Symposium, pp. 243-252, 1997.

[5] G. M. Tatchell, "Microbial insecticides and IPM: current and future opportunities for the use of biopesticides," in Microbial Insecticides: Novelty or Necessity? H. F. Evans, Ed., Proceedings of the British Crop Protection Council Symposium, 68, pp. 191200, 1997.

[6] H. F. Evans, "The role of microbial insecticides in forest pest management," in Microbial Insecticides: Novelty or Necessity? H. F. Evans, Ed., vol. 68 of Proceedings of the British Crop Protection Council Symposium, pp. 29-40, 1997.

[7] K. van Frankenhuyzen, R. C. Reardon, and N. R. Debois, "Forest defoliators," in Field Manual of Techniques in Invertebrate Pathology: Application and Evaluation of Pathogens for Control of Insects and Other Invertebrate Pests, L. A. Lacey and H. K. Kaya, Eds., pp. 767-785, Kluwer Academic Publishers, Dordrecht, The Netherlands, 2000.

[8] O. Skovmand, J. Kerwin, and L. A. Lacey, "Microbial control of mosquitoes and black flies," in Field Manual of Techniques in Invertebrate Pathology, L. A. Lacey and H. K. Kaya, Eds., pp. 767-785, Kluwer Academic, Dordrecht, The Netherlands, 2000.

[9] F. Fenner and F. N. Ratcliff, Myxomatosis, Cambridge University Press, Cambridge, UK, 1965.

[10] H. D. Burges and N. W. Hussey, Microbial Control of Insects and Mites, Academic Press, New York, NY, USA, 1971.

[11] R. M. Anderson and R. M. May, "Regulation and stability of host-Cparasite intercations. I. Regulatory Processes," Journal of Animal Ecology, vol. 47, pp. C219-C247, 1978.

[12] B. S. Goh, J. Leitman, and T. L. Vincent, "Optimal epidemic programs for pest control," in Management and Analysis of Biological Populations, B. S. Goh, Ed., 1980.

[13] P. Waltman and S. A. Levin, Deterministic Threshold Models in the Theory of Epidemics, Lecture Notes in Bioinformatics, Springer, Berlin, Germany, 1974.

[14] N. T. J. Bailey, The Mathematical Theory of Infectious Diseases and its Application, Griffin, London, UK, 1975.

[15] K. Wickwire, "Mathematical models for the control of pests and infectious diseases: a survey," Theoretical Population Biology, vol. 11, no. 2, pp. 182-238, 1977.

[16] H. R. Thieme, Mathematics in Population Biololgy, Princeton University Press, Princeton, NJ, USA, 2003.

[17] E. Kurstak, Microbial and Viral Pesticide, Marcel and Dekker, New York, NY, USA, 1982.

[18] M. B. Thomas, S. Blandford, C. Gbongboui, and C. J. Lomer, "Experimental studies to evaluate spray applications of a mycoinsecticide against the rice grasshopper, Hieroglyphus daganensis, in northern Benin," Entomologia Experimentalis et Applicata, vol. 87, no. 1, pp. 93-102, 1998. 
[19] A. B. Poore, "On the theory and application of the HopfFriedrichs bifurcation theory," Archive for Rational Mechanics and Analysis, vol. 60, no. 4, pp. 371-393, 1976.

[20] J. Guckenheimer and P. Holmes, Nonlinear Oscillations, Dynamical Systems, and Bifurcations of Vector Fields, vol. 42 of Applied Mathematical Sciences, Springer, New York, NY, USA, 1983.

[21] J. Hui and D. Zhu, "Dynamic complexities for prey-dependent consumption integrated pest management models with impulsive effects," Chaos, Solitons and Fractals, vol. 29, no. 1, pp. 233$251,2006$. 


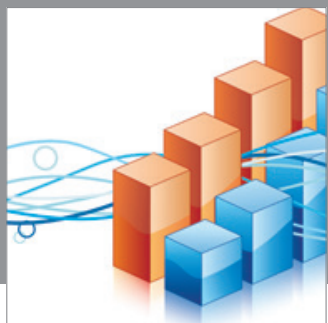

Advances in

Operations Research

mansans

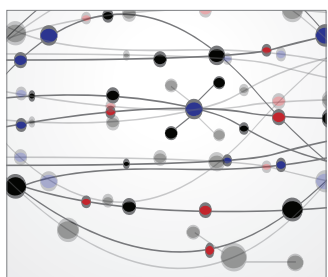

The Scientific World Journal
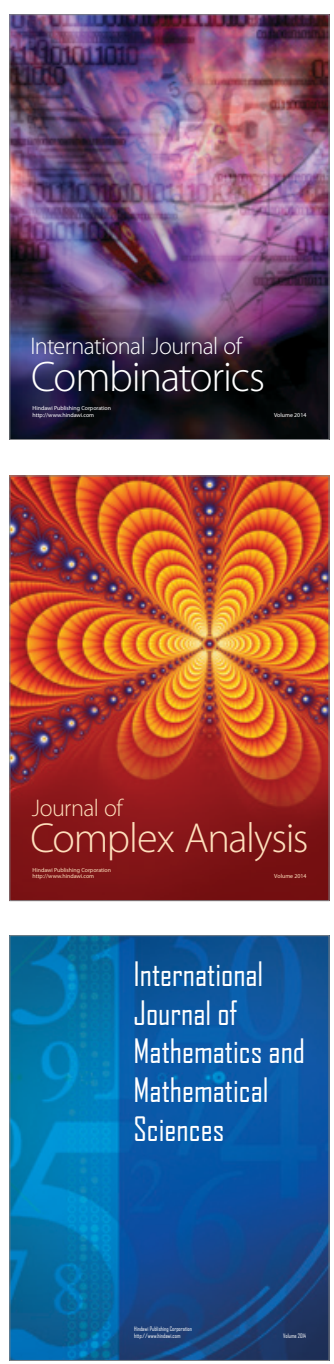
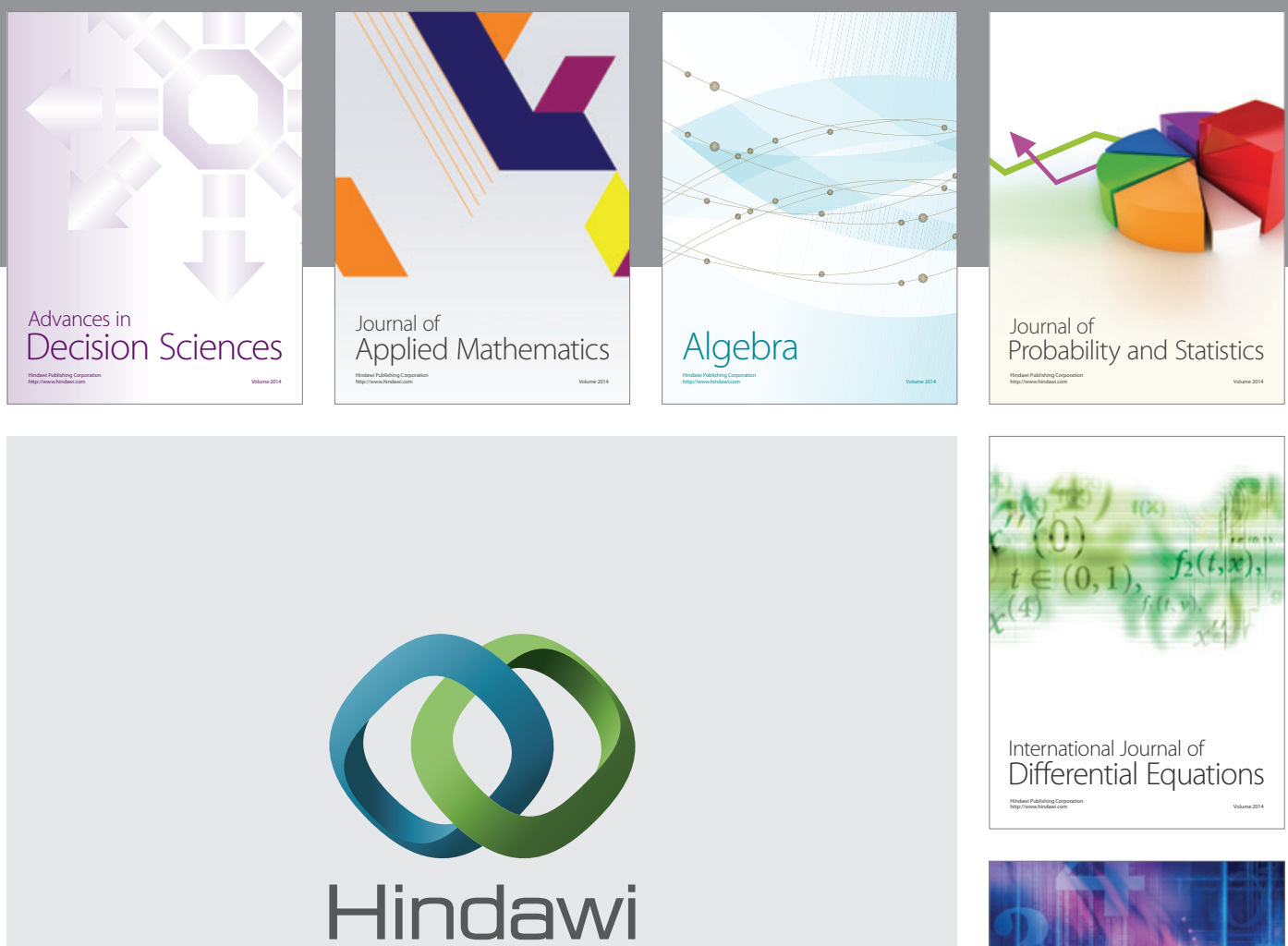

Submit your manuscripts at http://www.hindawi.com
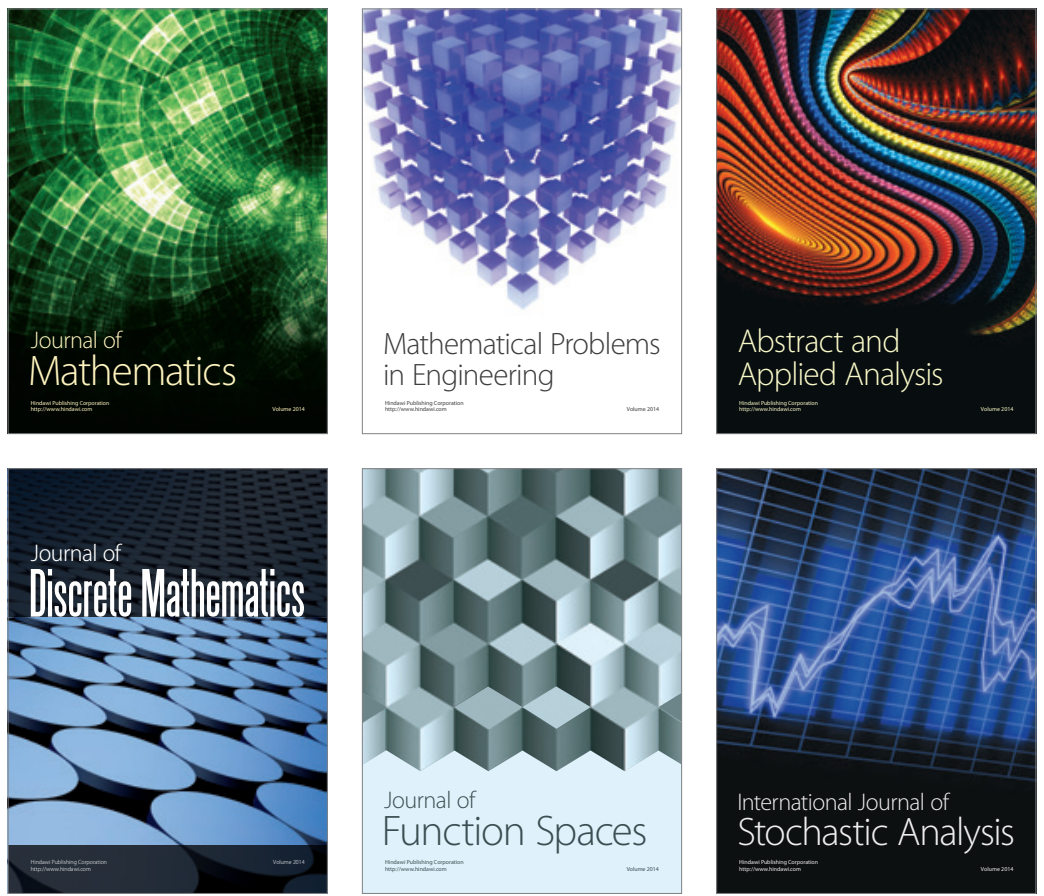

Journal of

Function Spaces

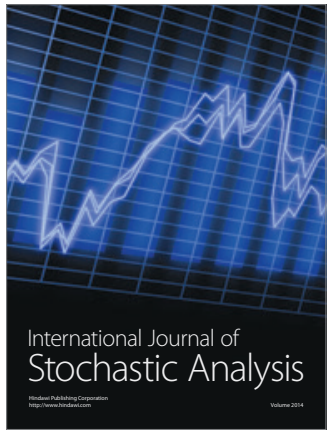

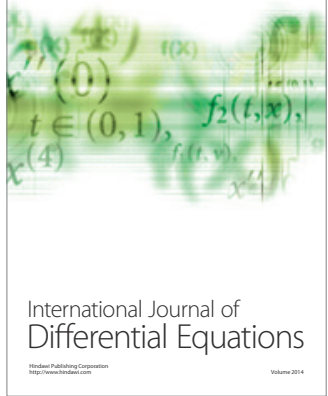
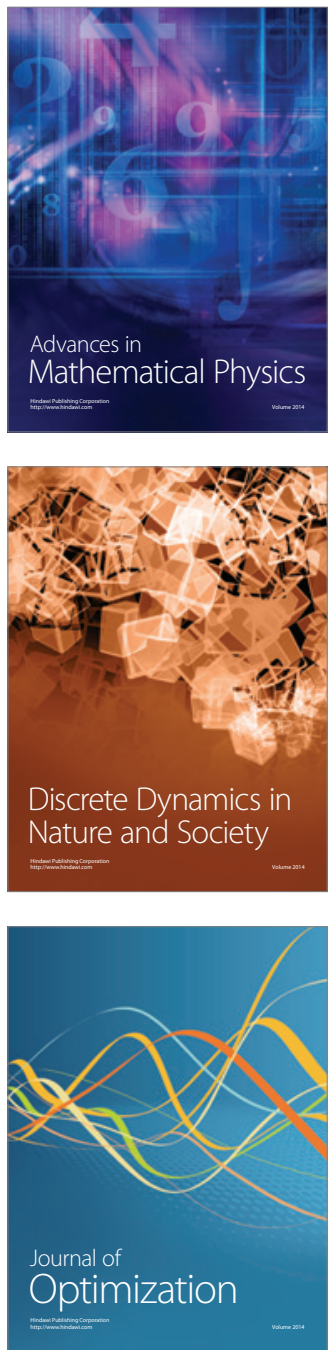